\title{
Shear Alfvén wave continuum spectrum with bifurcated helical core equilibria
}

To cite this article before publication: Allah Rakha et al 2019 Nucl. Fusion in press https://doi.org/10.1088/1741-4326/ab3a60

\section{Manuscript version: Accepted Manuscript}

Accepted Manuscript is "the version of the article accepted for publication including all changes made as a result of the peer review process, and which may also include the addition to the article by IOP Publishing of a header, an article ID, a cover sheet and/or an 'Accepted

Manuscript' watermark, but excluding any other editing, typesetting or other changes made by IOP Publishing and/or its licensors"

This Accepted Manuscript is @ EURATOM 2019.

During the embargo period (the 12 month period from the publication of the Version of Record of this article), the Accepted Manuscript is fully protected by copyright and cannot be reused or reposted elsewhere.

As the Version of Record of this article is going to be / has been published on a subscription basis, this Accepted Manuscript is available for reuse under a CC BY-NC-ND 3.0 licence after the 12 month embargo period.

After the embargo period, everyone is permitted to use copy and redistribute this article for non-commercial purposes only, provided that they adhere to all the terms of the licence https://creativecommons.org/licences/by-nc-nd/3.0

Although reasonable endeavours have been taken to obtain all necessary permissions from third parties to include their copyrighted content within this article, their full citation and copyright line may not be present in this Accepted Manuscript version. Before using any content from this article, please refer to the Version of Record on IOPscience once published for full citation and copyright details, as permissions will likely be required. All third party content is fully copyright protected, unless specifically stated otherwise in the figure caption in the Version of Record.

View the article online for updates and enhancements. 


\section{Shear Alfvén wave continuum spectrum with bifurcated helical core equilibria}

Allah Rakha ${ }^{1 *}$, Ph. Lauber ${ }^{2}$, M.J. Mantsinen ${ }^{1,3}$, D.A. Spong ${ }^{4}$

${ }^{1}$ Barcelona Supercomputing Center, 08034, Barcelona, Spain

${ }^{2}$ Max Planck Institute for Plasma Physics, Boltzmannstr. 285748 Garching, Germany

${ }^{3}$ ICREA, Pg. Lluís Companys 23, 08010 Barcelona, Spain

${ }^{4}$ Oak Ridge National Laboratory, TN, 37831, USA

E-mail: *allah.rakha@bsc.es

April 2019

Abstract. The radial structure of the continuum spectrum of shear Alfvén and Alfvén-acoustic waves in the beta-induced Alfvén eigenmode (BAE)frequency range is modeled for tokamak plasmas in the presence of 3D effects obtained from the bifurcated MHD equilibrium reconstruction. Plasma compressibility and geodesic curvature effects responsible for the low-frequency continuum spectrum calculations are invoked. In the equilibrium calculations we find that the helically distorted MHD equilibria may exist even for the axisymmetric devices if $\mathrm{q}=1$ rational surfaces are present. The continuum calculations with the bifurcated equilibria lead to a frequency splitting between the highest frequency branch and the lowest frequency branch continua at the frequency accumulation point. Radially localised shifting of modes happens via coupling of the adjacent n-1 continuum around an accumulation point. Our modelling (including 3D effects) correctly reproduces the phenomenon of continuum frequency splitting and provides a possible solution for the differences of few $\mathrm{kHz}$ in frequency splitting, which remained unexplained with the 2D kinetic calculations (Curran D. et al 2012 Plasma Phys. Control. Fusion 54 055001). The pressure scaling confirms the increase of helical excursion of the magnetic axis in equilibrium reconstruction and hence the range of continuum frequency splitting. In our calculations, the existence of low-frequency continua is in agreement with the experimentally observed low-frequency modes.

Keywords: Alfvén continuum, frequency splitting, helical core, 3D effects, tokamak

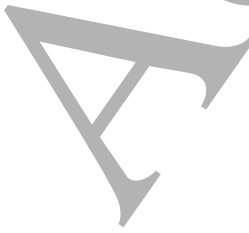

Submitted to: Nucl. Fusion 


\section{Helical Core paper}

\section{Introduction}

In fusion plasmas, fast ions in the $\mathrm{MeV}$ energy range may have velocities comparable with Alfvén velocity directed along the magnetic field lines, which can satisfy conditions of effective resonance and energy exchange between Alfvén waves [1]. Shear Alfvén Waves (SAW) form a continuous spectrum which in the presence of symmetry breaking non-uniformities such as toroidicity or ellipticity is modified to exhibit Alfvénic gaps. In this context two types of Alfvénic instabilities exist: (1) energetic particle continuum modes (EPMs) [2], dwelling in the Alfvén continuum and having frequencies in the range of the continuum and (2) discrete Alfvén eigenmodes (AEs) [3] with frequencies inside the SAW gaps. Given the strong continuum damping due to the collisionless dissipation of SAWs in non-uniform equilibria and their low absorption threshold for entering into an unstable state $[4,5]$, the question arises about the significance of continuum radial structures for the tokamak stability problem. Understanding the impact of the helical core on the modification of SAW continua may have potential implications for Alfvénic instabilities in burning plasmas and identify new issues in approaching the ignition margin.

In tokamak plasmas, the SAW continuum gets modified due to the interaction with low frequency MHD fluctuations, such as magnetic island and helical core. Previously, theoretical investigations for the Alfvén continuum have been made in the presence of magnetic islands [6]. In this paper, we have tried to investigate the effect of the helical core on the continuous spectrum. The helical core is an internal threedimensional (3D) structure in non-uniform axisymmetric MHD equilibria regarded as a saturated ideal internal kink mode and/ or a deformation of the magnetic axis. The helical excursion of the magnetic axis in a tokamak equilibrium appears because of the peaked pressure profile in the presence of a safety factor q passing through unity. The bifurcated equilibria are the combination of an axisymmetric part and a spontaneously triggered internal helical distortion of the magnetic axis, which are also called snakes in tokamaks [7] and in reversed field pinches (RFPs) single helical axis states (SHAx) [8]. The 3D helical deformation of the equilibrium destroys the symmetry properties of the tokamak. In/highly elongated plasmas, the disappearance of sawtooth oscillations and the persistence of a saturated ideal internal kink $\mathrm{m}=1, \mathrm{n}=1$ mode with $\mathrm{q}$ is in the vicinity of unity also can cause the existence of such helical deformations [9]. Plasmas with such special conditions are called hybrid plasma scenarios [10], which often trigger Alfvénic magnetohydrodynamic (MHD) instabilities such as EPMs [11], long-lived modes (LLMs) [12], and internal kinks or fishbones. Studies have shown that the 3D helical distortion during hybrid scenario operation is very large, which is confirmed by a complete flattening of strong NBI induced toroidal flow in the core region [13]. When the helical equilibrium is formed by an internal kink $\mathrm{m}=1, \mathrm{n}=1$ mode, the fast ions orbits become more complex with stonger radial drifts $[12,13]$.

In fusion plasmas, the sawtooth crashes are associated with the appearance of MHD instabilities such as saturated internal kinks [14] and continuous modes [15]. 


\section{Helical Core paper}

Studies have shown that the sawteeth disappear or transform into internal kink-like modes [14]. Observation of the low-frequency MHD modes during a sawtooth crash in ASDEX Upgrade (AUG) discharges has motivated employing saturated internal kinklike modes as helical distortions for further investigations of Alfvénic instabilities. AUG is a medium size divertor tokamak with a major and minor radius of 1.65 and 0.5 meters, respectively. At AUG, various ICRH-driven instabilities in the BAE and subBAE frequency range have been observed during monster sawtooth crashes [16, 17]. Understanding these instabilities, their impact on EP transport and their importance on sawtooth stabilisation is an outstanding issue in the view of predicting the sawtooth cycle properties in various ITER scenarios. In reference [17] a careful kinetic analysis has been carried out in order to understand the complex frequency evolution of these modes during a sawtooth cycle. Most of the aspects have been clarified such as the origin of the low $(\sim 10 \mathrm{kHz})$ and high $(\sim 70 \mathrm{kHz})$ frequency branches, the mode number inversion (higher toroidal mode numbers appear at lower frequencies) and the frequency evolution during the sawtooth cycle. The gyro-kinetic dispersion relation $[16,18,19]$ has been solved with the LIGKA code $[19,20]$ and compared to experimental measurements highlighting the importance of a kinetic analysis, in particular the role of diamagnetic effects [17]. However, 3D effects had not been included, and the question arises as to whether the presence of a helical core can modify the shear Alfvén continuum in a way to explain the remaining differences (see Fig. 7 and discussion at the end of section 2.4 of reference [17]).

So far, the effects of a helical core on calculations of Alfvén continuum have received very little attention. In our work, the helical core is modeled as a static helical distortion of the equilibrium because of its typicallow oscillation frequency as compared with the SAWs in AUG. Bifurcated tokamak equilibrium states are modeled using a 3D ideal MHD equilibrium solver VMEC [21] and it is generally found that the $\mathrm{m}=\mathrm{n}=1$ helical magnetic axis is displaced most at the position of $q_{\min }$ [12]. It is therefore clear that the Alfvén continuum needs to be modeled using a realistic 3D equilibrium magnetic field geometry. The shear Alfvén continuum is modeled by using an extended continuum solver (STELLGAP) $[22,23]$. STELLGAP has been previously used for the modelling of continuum structures in stellarators [24,25], RFPs [26] and tokamaks [27]. It is based on the reduced MHD model for 3D devices including effects of finite plasma compressibility and geodesic curvature. An equivalent set of reduced MHD equations for the low beta plasmas have been earlier used in similar tools COBRAS [28] and CONTI $[29,30]$ for the modelling of sound-Alfvén continua of 3D systems. Low frequency gaps in SAW continuum opened by the plasma compressibility and geodesic curvature are responsible for BAEs and beta-induced acoustic-Alfvén modes (BAAE). Coupling of the SAW with helical core distortion forms a linear model of SAW dynamics in a non-uniform equilibrium.

This paper is organized as follows. In section 2, the experimental details of the AUG discharges are provided. Section 3 provides the details of 3D MHD solver and in section 4 the extended Alfvén continuum solver STELLGAP is discussed. In section 5 


\section{Helical Core paper}

the details of the reconstruction of 3D helical core MHD equilibria are provided. Alfvén continuum calculations for the $2 \mathrm{D}$ and $3 \mathrm{D}$ equilibria are provided in section 6 and, finally, the summary and conclusions are provided in section 7 .

\section{Experimental Observations}

At the AUG tokamak experiments were conducted to investigate the low-frequency Alfvén instabilities. It has been observed that these modes appear during the sawtooth cycle and in the region surrounding with $q=1$ surface [16]. The appearance of lowfrequency modes around the region of $q=1$ during sawtooth cycle suggests possible helical core formation. In order to estimate the upper limit of the effect of the helical core, an AUG discharge \#20488 with monster sawtooth crashes is chosen. This discharge dates back to 2005 when the vessel wall was only $\sim 50 \%$ covered with tungsten $\mathrm{W}$ tiles. Similar discharges were repeated in 2009, however the sawtooth period was shorter and the pressure gradient at $\mathrm{q}=1$ before the sawtooth was slightly smaller, which has led us now to perform a pressure sensitivity scan in these calculations. In Figure 1, the BAE mode evolution in discharge \#20488 as measured with magnetic diagnostics is displayed. At this time high resolution Soft X-Rays (SXR) system was not available. In the case of discharge \#25546 the BAE and low-frequency modes measured with the core SXR channels are shown in Figure 2. The classification of the observed modes as BAEs and low-frequency modes and the splitting of several modes using the kinetic model has already been provided in [17]. The detailed experimental findings for the discharges \#20488 and \#25546 are published in [16] and [17] respectively.

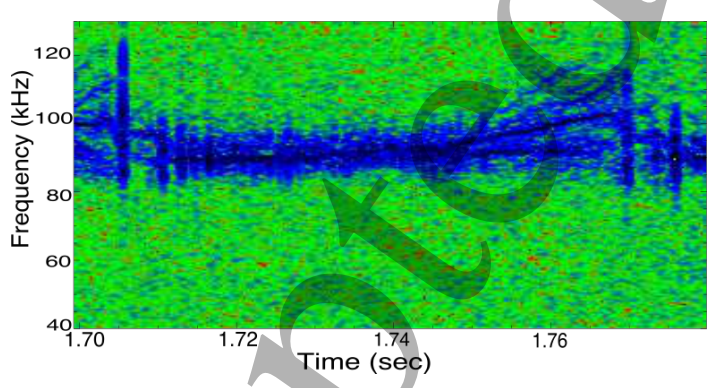

Figure 1: Frequency spectrogram for discharge \#20488 from $t=1.72$ to 1.78 seconds. Strong BAE mode activity during monster sawtooth crashes is measured with Mirnov coils.

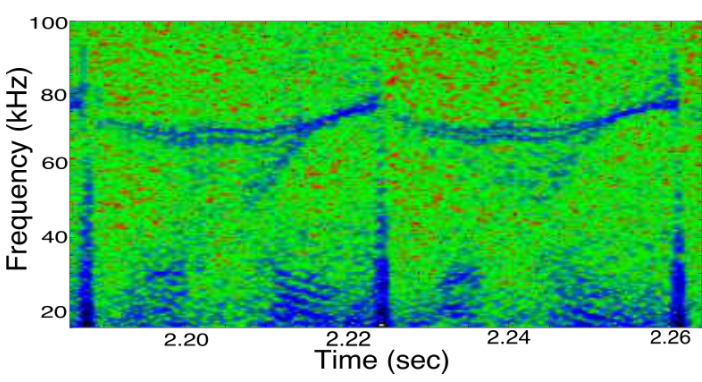

Figure 2: BAE and low-frequency mode activity for discharge \#25546 from $\mathrm{t}=2.0$ to 2.265 seconds measured with the core-localized soft X-ray channels.

In Fig. 1 of reference [17] the low-frequency mode activity during two sawtooth cycles from $\mathrm{t}=1.91$ to $1.98 \mathrm{~s}$ for discharge $\# 25546$ as measured with a core $(\rho \approx 0.24)$ soft $\mathrm{x}$-ray channel is shown. In these results $\rho$ is a normalized radial parameter defined as $\rho=\sqrt{\psi_{p o l}}$ and $\psi_{\text {pol }}$ is the normalized poloidal flux. Here, we focus on the highfrequency BAE branch at $\sim 70 \mathrm{kHz}$ with the toroidal mode numbers $\mathrm{n}=3$ to 6 . 


\section{Helical Core paper}

One can observe that the mode frequencies directly after a sawtooth crash are almost degenerated, whereas during the sawtooth cycle they start to split. This observation has been explained with the raise of the pressure gradient and the corresponding downshift of the kinetic continuum (see Fig. 12 of reference [17]). This implies that higher mode numbers are shifted downwards more strongly than lower mode numbers. Towards the end of the cycle, the mode-frequency splitting starts to disappear, probably due to the sawtooth precursors, as discussed in section 2.4 of reference [17]. Despite the success of this model, it is interesting to try to understand the remaining differences of 2 to $5 \mathrm{kHz}$ in frequency splitting, especially towards the end of the sawtooth cycle. It should be noted, that it is assumed that the evolution of the BAE continuum accumulation point is a proxy for the evolution of the global BAE mode [31]. Based on an ASDEX Upgrade equilibrium reconstruction for discharge \#20488 (and the practically identical discharge \#25546 discussed in [17]) a pressure scan is performed that is intended to model the increase in the core pressure after a sawtooth crash.

\section{3D MHD equilibrium solver VMEC}

In axisymmetric tokamak-like devices, the reconstruction of MHD equilibrium is usually performed in 2D formulation of the Grad-Shafranov equation. The 2D MHD equilibrium reconstruction sufficiently provides a basis for MHD stability studies unless the 3D structures are introduced in the calculations. External 3D perturbations in the magnetic field are introduced in tokamak plasmas to achieve optimized plasma performance [32]. In contrast to external perturbations, the internal 3D perturbations referred to as helical or snake equilibria, have also been studied for different tokamak devices i.e TCV [33], MAST [34], ASDEX-Upgrade [35], JET [7] and ITER [36]. These perturbations arise as the plasma self-organise to the lowest MHD energy state.

The mathematical description of the 3D MHD equilibrium reconstruction with a helical core using the 3D MHD equilibrium code VMEC [21] is based on the minimization of the plasma energy $W$. In this model, the nested magnetic flux surfaces are constrained to have a single magnetic axis, which preclude existence of magnetic islands and stochastic structures of the magnetic field. In VMEC the plasma energy is given by,

$$
W=\iiint d^{3} x\left(\frac{B^{2}}{2 \mu_{0}}+\frac{p_{\|}}{\Gamma-1}\right)
$$

where $B$ is the magnetic field strength, $\mu_{0}=4 \pi \times 10^{-7} \mathrm{H} / \mathrm{m}$ is the permeability of free space, $p_{\|}$is the parallel pressure, and $\Gamma$ is the adiabatic index. VMEC constructs an equilibrium by employing a variational method to find the minimum energy of the system. It solves an inverse equilibrium problem in cylindrical coordinates to determine the distance from the major axis $R=R(s, u, v)$ and height above the midplane $Z=Z(s, u, v)$, where $0 \leq s \leq 1$ is the radial variable corresponding to the normalized enclosed toroidal magnetic flux, $0 \leq u \leq 2 \pi$ denotes the poloidal angle and $0 \leq v \leq 2 \pi / N_{f p}$ is the toroidal angle where $N_{f p}$ is number of field periods around the torus. 


\section{Helical Core paper}

The variation of energy with respect to time is given as,

$$
\begin{aligned}
\frac{d W}{d t}=-\iiint d s d u d v & {\left[F_{R} \frac{\partial R}{\partial t}+F_{Z} \frac{\partial Z}{\partial t}+F_{\lambda} \frac{\partial \lambda}{\partial t}\right] } \\
& -\iint_{s=1} d u d v\left[R\left(p_{\perp}+\frac{B^{2}}{2 \mu_{0}}\right)\left(\frac{\partial R}{\partial u} \frac{\partial Z}{\partial t}-\frac{\partial Z}{\partial u} \frac{\partial R}{\partial t}\right)\right] .
\end{aligned}
$$

The last integral in Equation (2) defines the forces responsible for the movement of the plasma boundary. In fixed boundary calculations, the plasma-vacuum interface remains fixed. Therefore, by definition these forces vanish. The $R$ and $Z$ components of the MHD force are given $F_{R}=\sqrt{g} \nabla v \times \nabla Z \cdot F$ and $F_{Z}=\sqrt{g} \nabla R \times \nabla v \cdot F$ corresponding to the projection of the full force $\mathbf{F}=\mathbf{j} \times \mathbf{B}-\nabla \cdot \mathbf{P}$. The $\lambda$ force term is binormal (normal to the field line on a flux surface) force projection given as $F_{\lambda}=-\left(\sqrt{g} B \times \nabla s \cdot F / B^{2}\right)$, where $\lambda$ is poloidal angle re-normalization parameter, $\sqrt{g}$ is Jacobian and $p=p_{\|}=p_{\perp}$ is the isotropic pressure.

This system of MHD equations is solved iteratively by applying an accelerated steepest descent energy minimization scheme in VMEC. Fourier decomposition in the periodic angular variables $u$ and $v$ is used and a finite difference scheme is incorporated for the radial discretization. The radial force balance averaged over a flux surface is considered as diagnostic for the convergence of reconstructed equilibrium state. It is given by,

$$
\left\langle\frac{F_{s}}{\Phi^{\prime}(s)}\right\rangle=-\left\langle\left.\frac{1}{\Phi^{\prime}(s)} \frac{\partial p_{\|}}{\partial s}\right|_{B}\right\rangle-\frac{\partial}{\partial s}\left\langle\frac{\sigma B_{v}}{\sqrt{g}}\right\rangle-\iota(s) \frac{\partial}{\partial s}\left\langle\frac{\sigma B_{u}}{\sqrt{g}}\right\rangle
$$

where $\Phi$ is toroidal magnetic flux function, prime ( $I)$ denotes the derivative with respect to $s, \sigma=1 / \mu_{0}, B_{u}$ and $B_{v}$ are poloidal and toroidal magnetic field covariant components respectively and $F_{s}$ is covariant representation of the radial force, while $\iota(s)=1 / q$ the rotational transform of the magnetic field.

\section{The Alfvén continuum solver STELLGAP}

This section describes the mathematical model used for the calculation of Alfvén and Alfvén-acoustic continuum structures for three dimensional systems. The description of mathematical formulation is followed by the numerical scheme, employed in the extended STELLGAP solver.

To investigate the full continuum spectrum of ideal MHD, an extension of the STELLGAP solver [37] with slow-sound wave coupling [38] has been used. Computation of the Alfvén continua with the coupling of slow-sound and Alfvén waves is viewed as obligatory due to the low frequency oscillations in the experimental observations as discussed in section 2. Three dimensional reduced MHD equations with the inclusion of parallel compressibility and coupling to Alfvén and magneto-acoustic waves for low beta toroidal plasmas have been derived [39] for finite geodesic curvature of the magnetic 


\section{Helical Core paper}

field lines. The curvature $K_{s}$ of field lines is responsible for coupling between these two waves, since at vanishing $K_{s}$ the two waves decouple.

The inclusion of parallel compressibility into the system of equations allows us to investigate, in addition to the shear Alfvén mode, the acoustic, geodesic acoustic and coupled acoustic-shear Alfvén modes. These effects of compressibility are expected to set a minimum frequency range at rational surfaces $\left(k_{\|}=0\right)$ for the continuum. Following the approach of [40], the derived system of equations [39] is transformed into an eigenvalue system in the extended STELLGAP code $[22,23]$. This eigenvalue system can be written as,

$$
\begin{gathered}
\left(\mu_{0} \rho_{m} \omega^{2} \frac{|\vec{\nabla} \psi|^{2}}{B^{2}}+\vec{B} \cdot \vec{\nabla}\left[\frac{|\vec{\nabla} \psi|^{2}(\vec{B} \cdot \vec{\nabla})}{B^{2}}\right]\right) \xi_{s}+\gamma p K_{s}(\vec{\nabla} \cdot \vec{\xi})=0 \\
K_{s} \xi_{s}+\left[\frac{\gamma p+B^{2}}{B^{2}}+\frac{1}{\mu_{0} \rho_{m} \omega^{2}} \gamma p(\vec{B} \cdot \vec{\nabla}) \frac{(\vec{B} \cdot \vec{\nabla})}{B^{2}}\right](\vec{\nabla} \cdot \vec{\xi})=0
\end{gathered}
$$

In both equations, $(\vec{\nabla} \cdot \vec{\xi})$ is the plasma compressibility, magnetic surface displacement is $\xi_{s}=\vec{\xi} \cdot(\vec{B} \times \vec{\nabla} \psi) /|\nabla \psi|^{2}$ and $\vec{\nabla} \psi=N_{f p} F_{p}^{\prime} / 2 \pi \vec{\nabla} s$, where $\psi$ is the toroidal flux, $N_{f p}$ is field-period and $F_{p}^{\prime}$ is derivative of the poloidal flux with respect to normalized toroidal flux $s$ per period. $K_{s}$ is the geodesic curvature of the background magnetic field given by,

$$
K_{s}=2 \vec{\kappa} \cdot\left(\vec{B} \times \frac{\vec{\nabla} \psi}{B^{2}}\right) \text { with } \vec{\kappa}=(\vec{b} \cdot \vec{\nabla}) \vec{b} \quad \text { and } \quad \vec{b}=\frac{\vec{B}}{B}
$$

Equations (4) and (5) are solved as a coupled system, expanding the unknowns in a Fourier series in poloidal $(\mathrm{m})$ and toroidal $(\mathrm{n})$ mode numbers. This leads to a matrix eigenvalue equation:

$$
\omega^{2} \overleftrightarrow{A} \vec{x}=\overleftrightarrow{B} \vec{x}
$$

where $\vec{x}$ is a vector containing the Fourier components of $\xi_{s}$ and $\vec{\nabla} \cdot \vec{\xi}$. With this set of equations, the Alfvén and Alfvén-sound continuum is computed by STELLGAP for all relevant mode numbers $(\mathrm{m}, \mathrm{n})$ using the DGEGV routine from the LAPACK library.

Equation (7) is solved in straight field line Boozer coordinates. The use of straight field line coordinates preserves the zeros of the B $\cdot \nabla$ operator when coupled with a Fourier series representation of $\vec{\nabla} \cdot \vec{\xi}$ to avoid the presence of singularities due to the compressibility effects. The unknowns $\xi_{s}$ and $\vec{\nabla} \cdot \vec{\xi}$ are expanded in a Fourier series over poloidal and toroidal angles, along with the coefficients in Equations (4) and (5). Series resulting from the convolution of these product series is multiplied by the individual Fourier test functions and integrated over each flux surface. This results in block tridiagonal matrices with the block size equal to the number of Fourier modes. An eigenvalue problem is then solved for each flux surface. A wide range of the Fourier modes is implemented to cover the extended spectrum of shear Alfvén, acoustic and coupled acoustic-shear Alfvén continua. 


\section{Helical Core paper}

\section{Bifurcated MHD equilibrium reconstruction}

The saturated internal-kink mode is dominated by $\mathrm{m}=1, \mathrm{n}=1$, surrounding the magnetic axis and leads to the helical equilibria. They distort the symmetry properties in the plasma core area by forming a 3D helical core surrounded by an axisymmetric. 2D mantle. Plasma equilibria with a 3D helical core and axisymmetric boundary are defined as bifurcated helical equilibrium states.

In our studies, we have modeled a bifurcated helical equilibrium by introducing a small perturbation in the magnetic axis for AUG-tokamak discharge \#20488, which has $q$ in the vicinity of unity in the core region of the tokamak. Two sister equilibria, one axisymmetric and another helical are calculated simultaneously using an identical boundary with the VMEC code [21]. The q-profile of the discharge \#20488 presented in Figure 3 is used as an input to the VMEC code together with the plasma boundary, peaked pressure profile (Figure 4) and an initial perturbation in the magnetic axis. The q-profile in Figure 3 shows the extended region of low shear up to $\rho=0.4$ and q close to one. These are the principal requirements for the formation of a helical core.

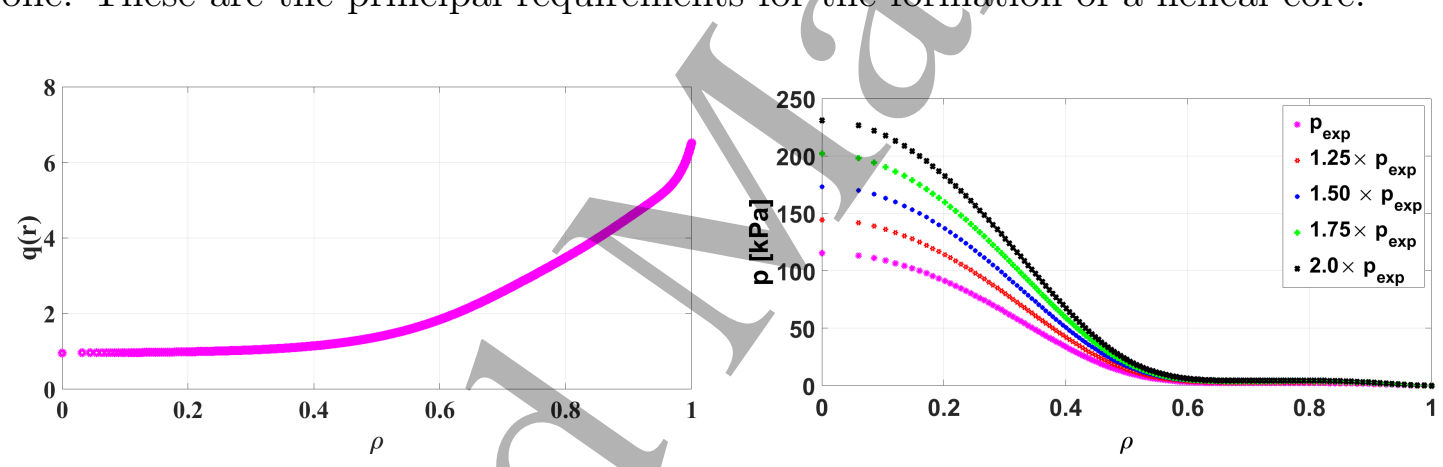

Figure 3: Safety factor (q) profile of Figure 4: Plasma pressure profile ASDEX-Upgrade discharge \#20488. of AUG discharge \#20488. Here

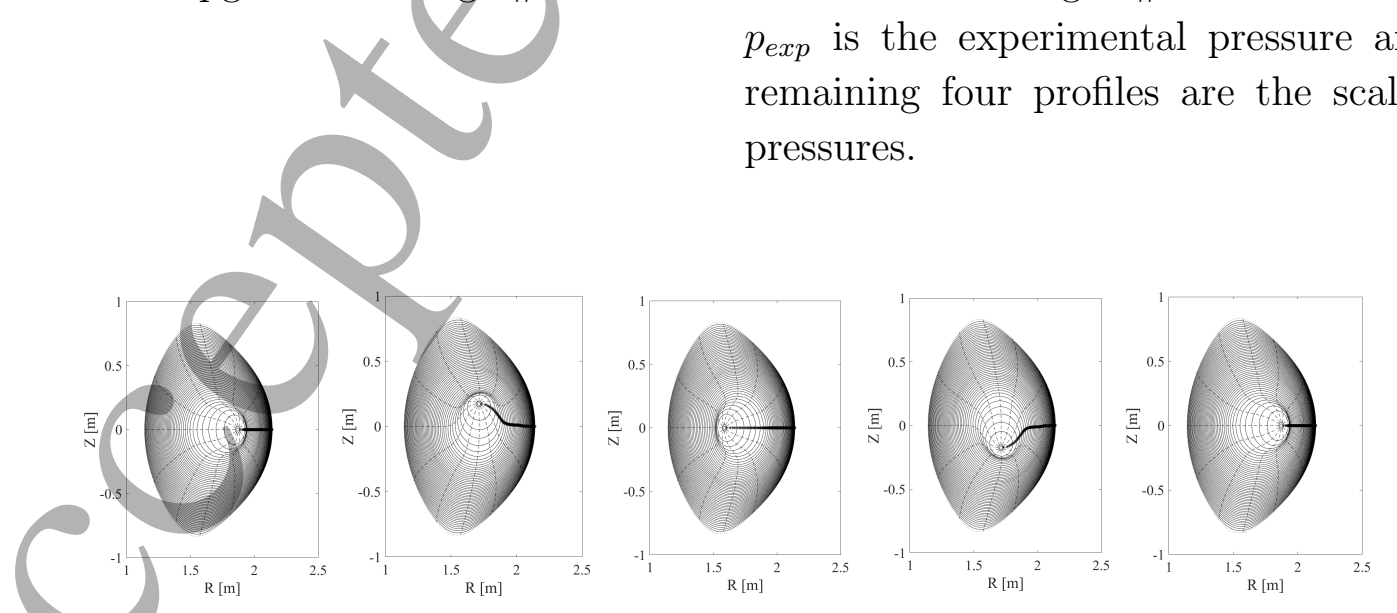

Figure 5: Poloidal cross-section of the toroidal magnetic flux surfaces for helical core equilibria with $\delta_{H}=0.37$ at five toroidal angles $\phi=0, \pi / 2, \pi, 3 \pi / 2,2 \pi$ (left to right). The deformations in flux surfaces are helical excursions of the bifurcated equilibrium while preserving the plasma boundary 


\section{Helical Core paper}

The reconstructed equilibrium having the bifurcated behavior can be assessed through the progression of modified magnetic flux surfaces. The magnetic flux surfaces are plotted in the Figure 5, which clearly shows modifications in those flux surfaces around the torus, which are clear signals of the helical core formation near the magnetic axis. The saturated state of the magnetic axis can be characterized as an $\mathrm{m}=1$ and $\mathrm{n}=1$ perturbation, the internal kink mode. It can be seen that while qualitatively the helical formation is similar, it changes its orientation along the toroidal angle. In comparison with these flux shape variations, the axisymmetric equilibrium calculations display continuous magnetic flux surfaces with no deformation across the whole torus as shown in Figure 6. In these calculations, identical pressure, inverse rotational transform (q-profile) and plasma boundary are imposed. The only difference is the removal of the seed perturbation of the magnetic axis, which is added for the helical core calculations to allow the magnetic axis transform to a saturated internal-kink mode, if necessary conditions permit [33].

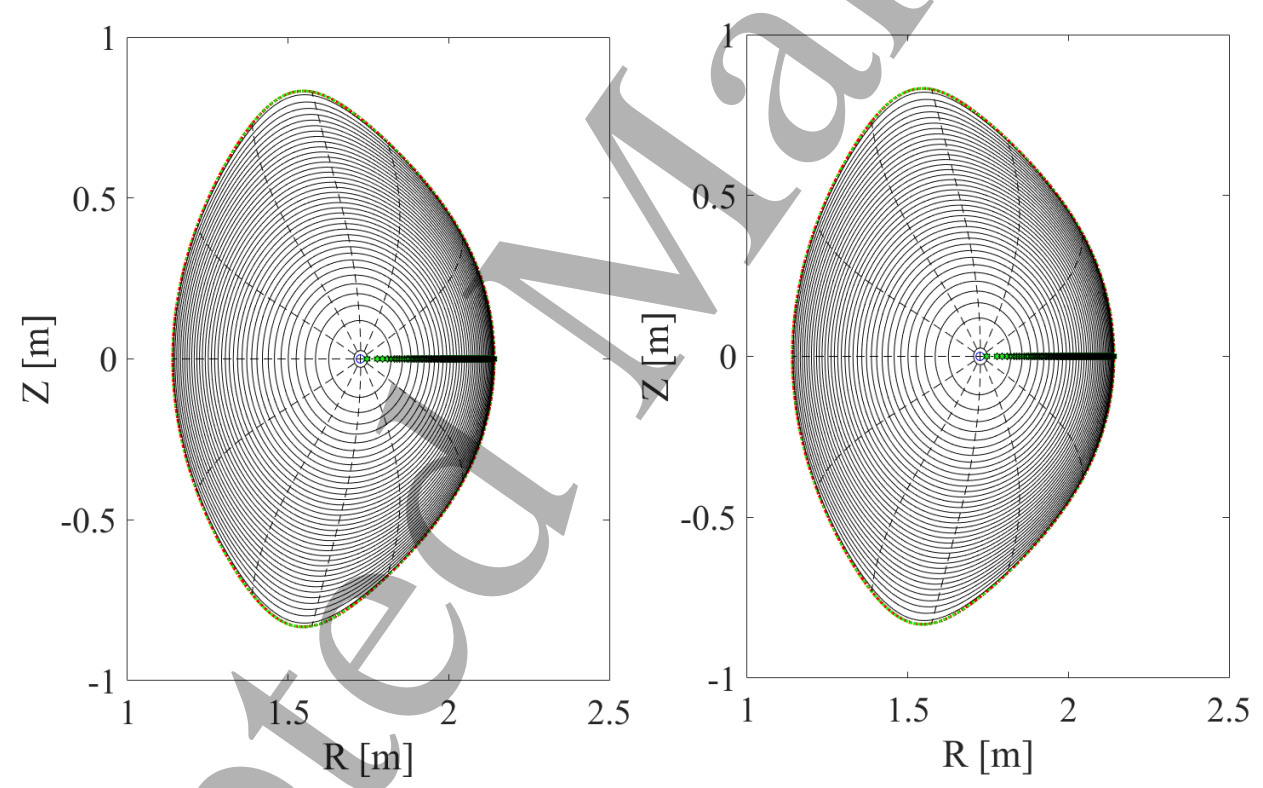

Figure 6: Poloidal cross-section of the magnetic flux surfaces at two different toroidal angles $\phi=0, \pi / 2$ and $\pi / 2, \pi$ for the axisymmetric equilibria. In axisymmetric equilibrium both contours have exactly matching flux surfaces and the same magnetic axis. Here the magnetic axis is represented with blue and green colors.

In the comparison of magnetic flux surfaces contours at two different toroidal angles, the results for axisymmetric case are shown in Figure 6, which confirm the zero level of distortion in the flux surfaces. On the other hand, the 3D helical core comparison given in Figure 7 for the two identical toroidal angles provides the proof of relative excursion of magnetic axis due to helical core formation and simultaneous existence of two equilibria solutions, one with helical equilibrium in plasma core and second with axisymmetric equilibrium in the rest of contour are confirmed. This comparison shows the qualitative excursion of the magnetic axis at two different toroidal angles and highlights the regions 

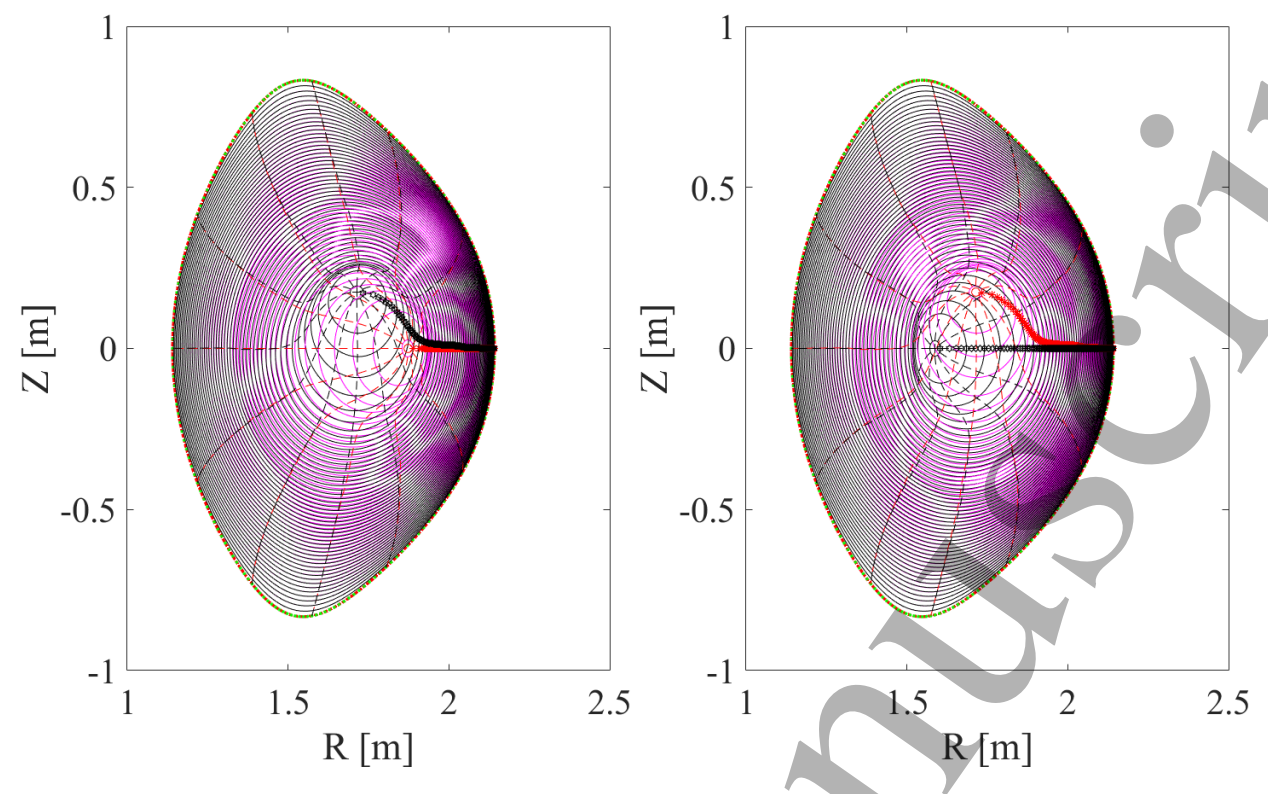

Figure 7: Poloidal cross-section of the magnetic flux surfaces at two different toroidal angles $\phi=0, \pi / 2$ and $\pi / 2, \pi$ for the helical core equilibria with $\delta_{H}=0.37$. In both graphs, the excursion of the magnetic axis is clear and represented by red and black symbols and the magnetic flux surfaces are represented by magenta and black color lines. The red and black color dashed lines are the theta contours.

of closely packed magnetic flux surfaces due to the helical core formation.

The convergence of the reconstructed equilibria has been investigated by using the volume averaged residual horizontal force $F_{R}$ as a function of number of iterations as shown in Figure 8. The significant drop in $F_{R}$ along the number of iterations confirms the convergence of helical core formation results. As in previous investigations [7], the normalized radial force balance given in the Equation (3) has been used as a diagnostics for the convergence of the equilibrium state by reaching to the value of the order of $10^{-8}$. In our results, with the number of flux surfaces equal to 799 , we have achieved a similar level of convergence in around 20000 iterations for the experimental pressure. In the higher pressure calculations, we have used around 40000 iterations to achieve the converged results for 500 radial grid points.

We have also modeled the excursion of the helical core $\delta_{H}$ defined in Equation (8) with the number of iterations. It is found that the helical core saturates at an identical number of iterations for different radial resolutions. In previous studies the saturation of the helical core with a higher number of radial grid points [41] and the increase in the helical core with the increase in number of iterations [35] has been associated with the convergence of helical core equilibria. In our results presented in Figure 9, the helical core for the lower number of iterations remains smaller, and after reaching a sufficient number of iterations as defined by the threshold given in [41], it grows rapidly and eventually saturates. At this point the helical core becomes fully resolved.

The quantitative measure of the helical excursion for the reconstructed equilibria 
1

2

3

4

5

6

7

8

\section{Helical Core paper}

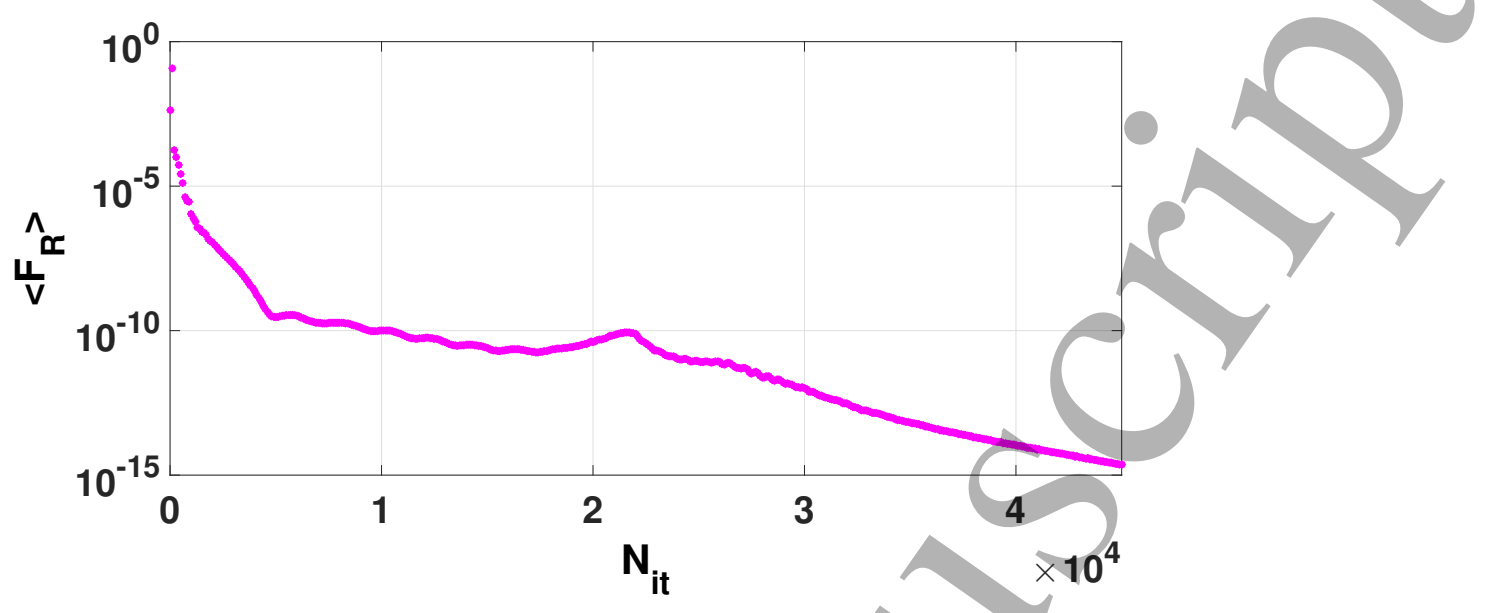

Figure 8: The volume averaged horizontal force $F_{R}$ evolution with number of iterations

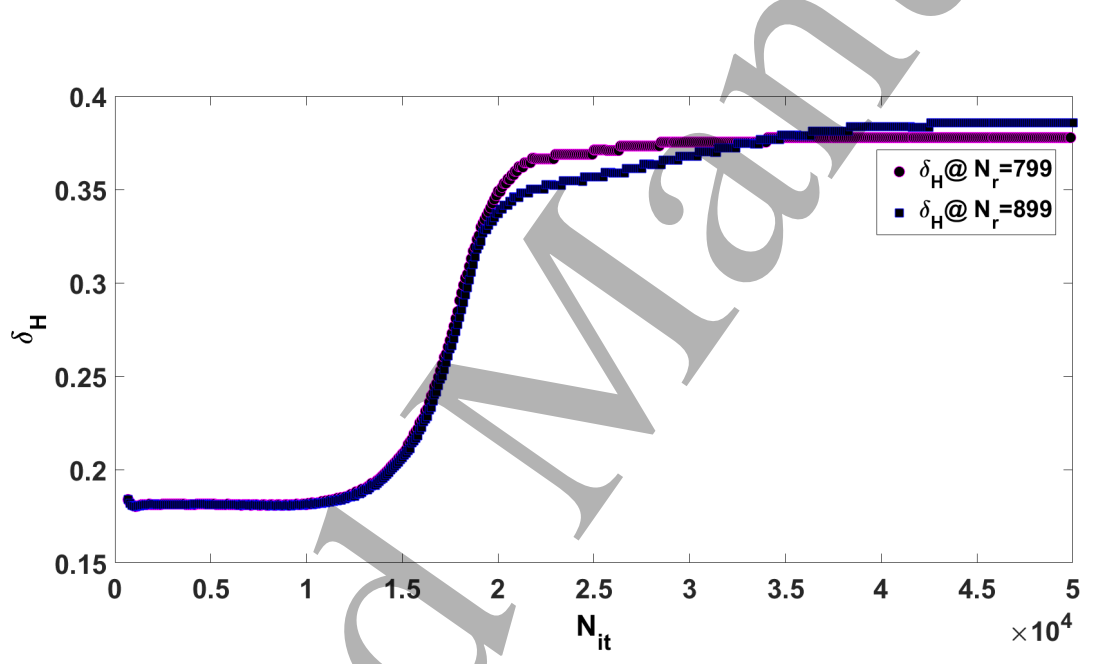

Figure 9: Dependence of helical core size $\delta_{H}$ on number of iterations $N_{i t}$ for different levels of radial resolution $N_{r}$

can be provided with a parameter $\delta_{H}[7]$ defined as

$$
\delta_{H}=\frac{\sqrt{R^{2}{ }_{01}(s=0)+Z^{2}{ }_{01}(s=0)}}{a}
$$

Here $R_{01}\left(Z_{01}\right)$ corresponds to the Fourier amplitude of the $R(Z)$ components of the $m=0, n=1$ mode at the magnetic axis $s=0(a=0.4$ meters is the effective plasma minor radius).

In this work the helical excursion of the magnetic axis has been calculated with respect to the plasma pressure as shown in Figure 10, as the peaked plasma pressure has been considered an important parameter in the formation of helical structures. Results computed with fixed boundary equilibrium calculations, show that the helical excursion of the magnetic axis increases with the increase in the plasma pressure up to a certain limit, from $\delta_{H}=0.37$ for the experimental pressure to $\delta_{H}=0.7$ for 1.75 times of the experimental plasma pressure. When the plasma pressure is increased further, the helical 


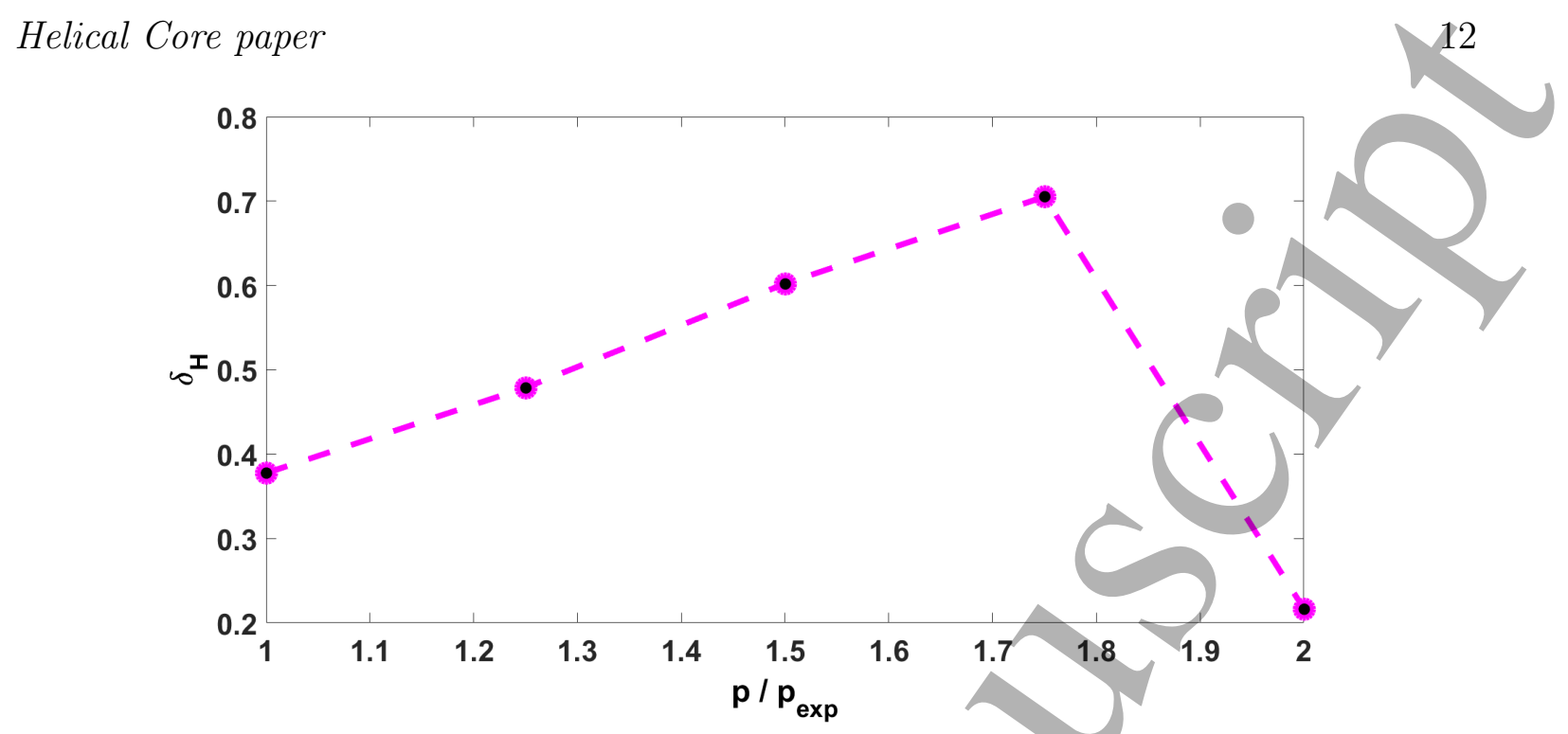

Figure 10: The magnetic axis helical distortion parameter $\delta_{H}$ as a function of the pressure normalized to the experimental pressure.

excursion decreases due to the fixed boundary nature of the equilibrium calculations. In a fixed boundary equilibrium model, the Shafranov shift at the maximum plasma pressure already distorts the magnetic flux surfaces strongly enough and leaves no room for their further stretching. Therefore, a further excursion of the helical core is not possible at the higher plasma pressures.

\section{Alfvén continuum results and discussions}

Alfvén continuum calculations have been performed for the experimentally observed radially extended low-shear q-profile plasmas using axisymmetric and non-axisymmetric MHD equilibria as discussed in section 5. Two different cases, one with axisymmetric (2D) and the second one with helical (3D) equilibria have been investigated. In Alfvén continuum calculations the parallel plasma compressibility $\gamma=1.6$ is employed for the low frequency Alfvén continuum calculations. In these calculations, we have limited the selection of toroidal mode numbers $(n)$ from 1 to 6 , since a similar range of $n$ was measured in the experiments.

\subsection{Alfvén continuum calculations with axisymmetric 2D equilibria}

Axisymmetric equilibrium calculations for tokamaks are straightforward because ideal tokamaks are inherently axisymmetric (2D) devices. Alfvén continua using an axisymmetric equilibrium are calculated for $\mathrm{n}=1$ to 6 . The Alfvén continuum structures for the full radial extent and frequencies up to $475 \mathrm{kHz}$ are shown in Figure 11. In these continuum structures a frequency gap appears in the full plasma column. The gap is wide in the core and becomes narrower towards the edge. These structures are similar for all toroidal mode numbers, including their resonant extrema points. 
For the six toroidal mode numbers $n=1$ to 6 considered here, the Alfvén continuum structures at $\rho \approx 0.23$ approach an accumulation point as shown in Figure 11 around the $\mathrm{q}=1$ rational surface exhibited in Figure 3. Zooming in on these structures in Figure 12 shows that all continua accumulate around a single frequency of $35 \mathrm{kHz}$ and there is no splitting in the frequency range. These characteristics of non-splitting frequency at the accumulation point around rational surfaces are the hallmarks of axisymmetric continua. Here, we define frequency splitting $(\Delta f)$ as the difference between the highest frequency branch and the lowest frequency branch in the continuum structures around the frequency accumulation point caused by the 3D effect of the helical core. We will study this frequency splitting in detail in section 6.2 below.

It should be noted that in the experiment the BAE mode frequency was observed at $60-70 \mathrm{kHz}$. The remaining difference after taking into account the Doppler shift $\sim$ $3 \mathrm{kHz}$ [17] can be attributed to a different model for the plasma compressibility: here the value of $\gamma=1.6$ is used where as in [17] the more sophisticated kinetic expression was used. Nevertheless, the frequency splitting due to 3D effects is not expected to change significantly due to the choice of this parameter.

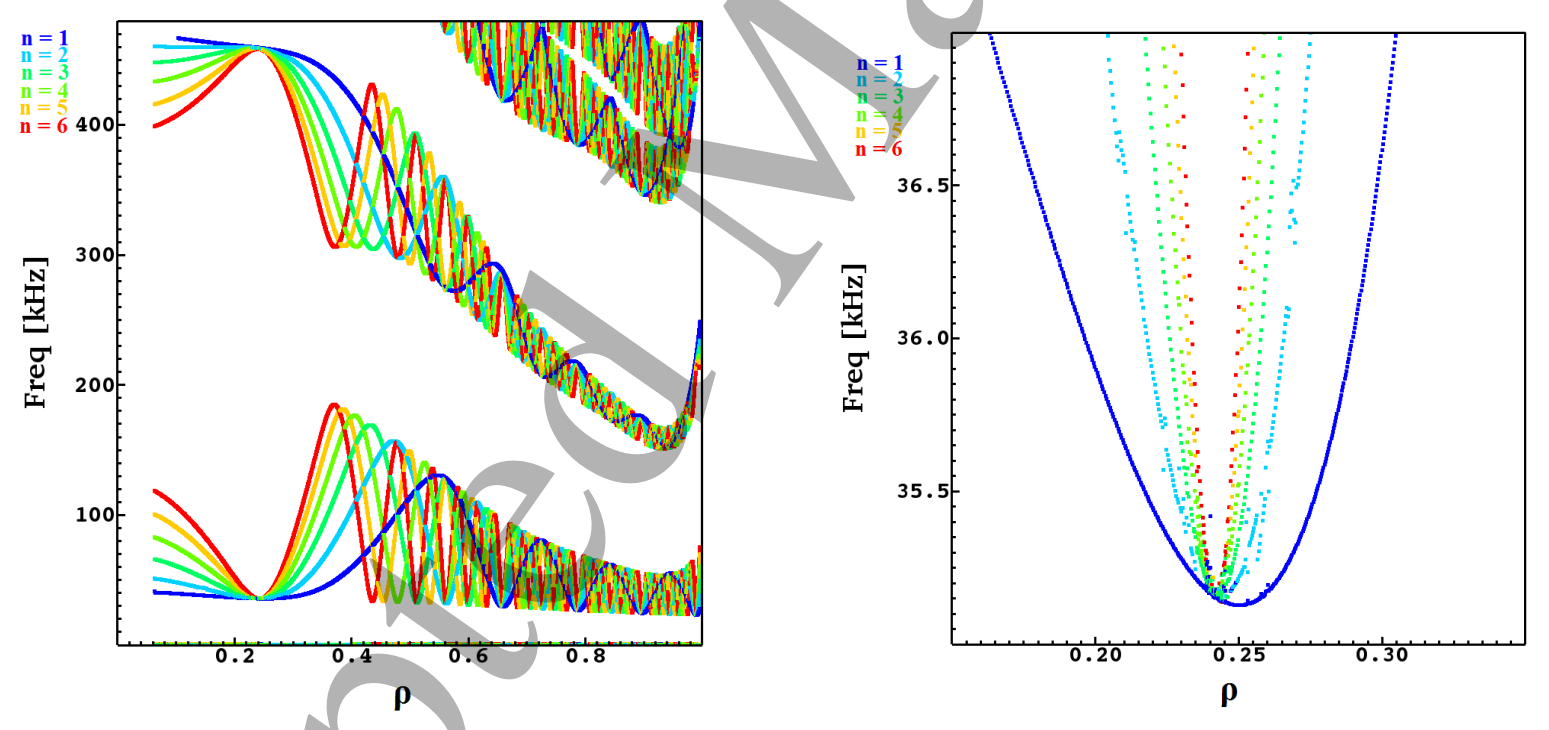

Figure 11: Alfvén continuum structures with axisymmetric (2D) equilibrium for discharge \#20488.
Figure 12: Zoom in of Alfvén continua in Figure 11. Different colors represent the corresponding toroidal modes number $(n)$

\subsection{Alfvén continuum calculations with bifurcated helical core equilibria}

The continuum structures using helical 3D equilibria have been investigated for the same discharge for the five different pressures (Figure 4) as discussed in the section 5. The helical core excursion shown in Figure 10 is strongly dependent upon the plasma pressure. The Alfvén continuum calculations are performed to investigate the effect 


\section{Helical Core paper}

of helical core size, since in the experimental investigations a peaked pressure profile was measured after the sawtooth crashes. Simulation results of Alfvén continua for the experimental pressure with an $\mathrm{m}=1, \mathrm{n}=1$ helical core and $\delta_{H}=0.37$ are presented in Figures 13 and 14. For the maximum achieved helical core size of $\delta_{H}=0.7$ the Alfvén continua are presented in Figures 15 and 16.

There are a number of significant differences in the Alfvén continua computed using bifurcated MHD equilibria with the Alfvén continua computed using an axisymmetric equilibrium. They include: frequency splitting in Alfvén continua, shifting of the modes at the frequency accumulation point and the appearance of helical Alfvén eigenmodes (HAE) gaps. In following, we discuss the differences in detail.

Alfvén continua computed using helical equilibrium and having exactly equivalent plasma parameters as the axisymmetric calculations is shown in Figure 13. Around the frequency accumulation point at $\rho \approx 0.23$ there is a noticeable splitting in the frequency range of continua as shown in Figure 14. Following the definition of frequency splitting, we have the highest frequency branch at $\mathrm{f}=36.4 \mathrm{kHz}$ with toroidal mode number $\mathrm{n}=$ 5 and the lowest frequency branch at $\mathrm{f}=33.4 \mathrm{kHz}$ with toroidal mode number $\mathrm{n}=6$, resulting in $\Delta f=3 \mathrm{kHz}$. The radial shifting in the mode structures via coupling to the $\mathrm{n}-1$ continuum structures around an accumulation point is also clearly shown in Figure 14 e.g. a mode $\mathrm{n}=1$ converts to an $\mathrm{n}=6$ mode in the range $0.218 \leq \rho \leq 0.238$, then it reverts back to a $n=1$ mode at higher $\rho$.

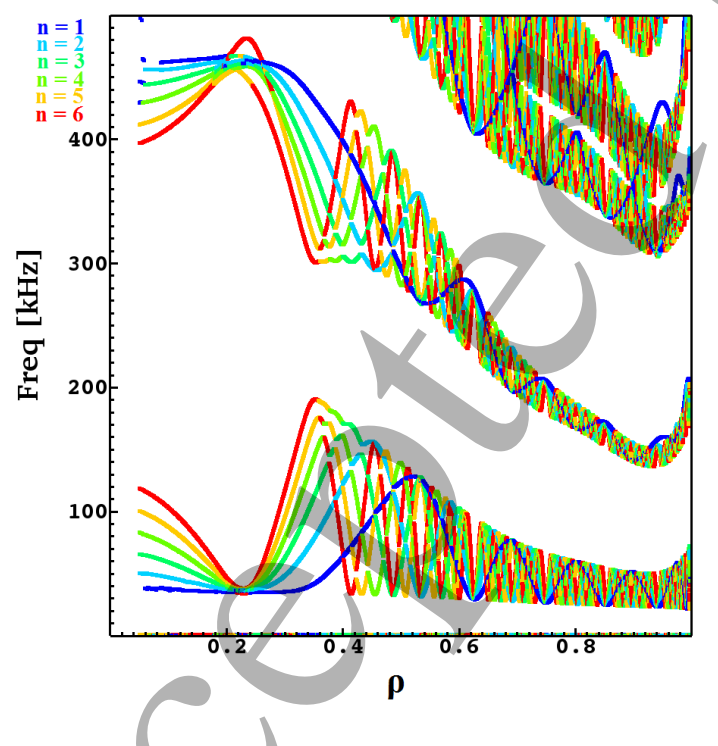

Figure 13: Alfvén continuum structures with $3 \mathrm{D}$ helical core equilibrium having $\delta_{H}=0.37$ for discharge \#20488

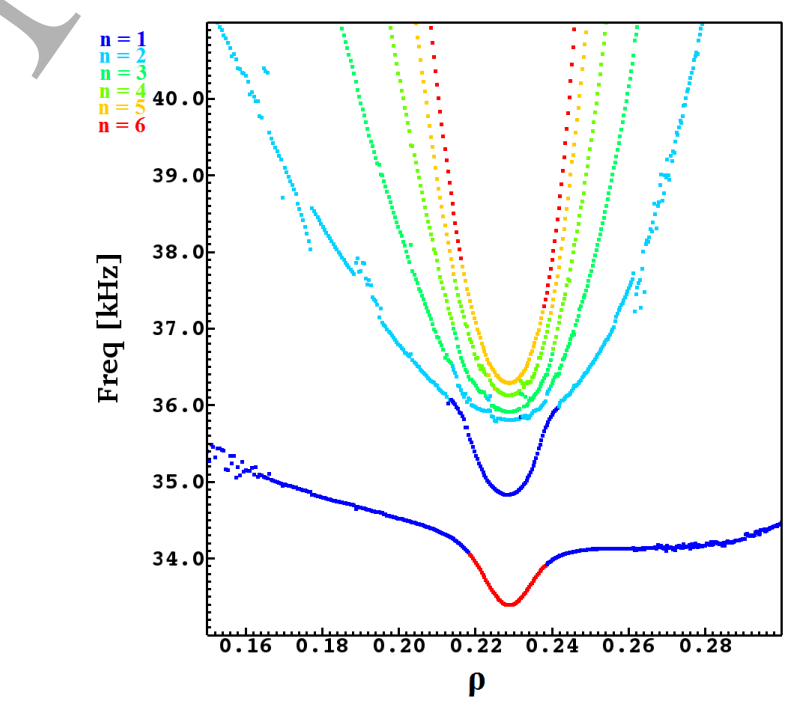

Figure 14: Zoom of Alfvén continua in Figure 13. Different colors represent the corresponding toroidal modes number (n)

The radial shifting in mode structures is associated with the change in $k_{\|}$due to the $3 \mathrm{D}$ excursion of magnetic axis. Due to the helical contribution, for the small $k_{\|}$ at the accumulation point, the smaller non-axisymmetric sideband terms compete with 
the axisymmetric terms and become prominent. Close to $k_{\|}=0$ they become more significant. This prominence of non-axisymmetric sidebands at smaller $k_{\|}$values leads to a radial shifting of modes around the accumulation point.

In figure 7 of [17], the shifting of the Alfvén continua relative to each other have also been modeled using a kinetic Alfvén model. They observed that the modes with higher $n$ were shifted downwards after a few seconds of observation due to the strong diamagnetic frequency. Similarly, we have computed the shifts in Alfvén/continua around the frequency accumulation point with the formation of helical core equilibria with a saturated 3D magnetic axis.

For the extreme helical excursion of $\delta_{H}=0.7$, which happens at 1.75 times the experimental pressure, the computed Alfvén continuum structures are presented in Figure 15. In this case, the frequency gap around the plasma axis decreases by $20 \mathrm{kHz}$ as compared with the Alfvén continuum calculated at the experimental pressure and helical core equilibria (Figure 13). The maximum frequency of the Alfvén continuum drops to $410 \mathrm{kHz}$ from $475 \mathrm{kHz}$ with minimum of $32 \mathrm{kHz}$. The splitting in the frequency range around the accumulation point $\rho \approx 0.23$ has also increased to $5 \mathrm{kHz}$, which corresponds to the difference between thethe highest frequency branch $\mathrm{f}=32.6 \mathrm{kHz}$ with toroidal mode number $\mathrm{n}=4$ and the lowest frequency branch $\mathrm{f}=27.6 \mathrm{kHz}$ with toroidal mode number $\mathrm{n}=1$ as shown in Figure 16. In our analysis, for lower $\left(\delta_{H}=0.37\right)$ as shown in figure 14 , the continuum of each $n$ is shifted with the adjacent lower continuum of $n-1$ around the frequency accumulation point. Only the continuum of $n=1$ changes with the continuum of $n=6$ due to our selection of a maximum toroidal mode of $\mathrm{n}=6$ in the STELLGAP code. In figure 16 with strong helical deformation $\left(\delta_{H}=0.7\right)$ this type of mode transitioning around the accumulation point from $\mathrm{n}$ to $\mathrm{n}-1$ no longer holds, this can be due to the stronger helical distortion resulting in further radial stretching of the accumulation point. The radial drift of the accumulation point is associated with the increase in the Shafranov shift due to the higher plasma pressures [42].

Coexistence of modes with similar $n$ over a significant radial extent is associated with the existence of radially extended rational surfaces and the saturation level of the helical excursion of the magnetic axis. These factors mutually tend to maintain smaller $k_{\|}$around the radially localized accumulation point; additionally for higher $\delta_{H}$ the frequency accumulation point becomes radially extended as seen in figure 16 . As mentioned earlier, under these conditions the frequency shifting phenomenon and prominence of the non-axisymmetric sidebands undergo the radial transition of the modes which leads to the coexistence of modes over a wide radial extent since the accumulation point at stronger $\delta_{H}$ gets stretched in radius. The successive transition of the modes happens as shown in figure 16 , in the mode $\mathrm{n}=2$ branch which converts into a $\mathrm{n}=1$ mode in the range $0.205 \leq \rho \leq 0.236$, and beyond $\rho \approx 0.236$ it again changes into an $n=3$ mode. Later it transforms back to $\mathrm{n}=1$ and finally reverts back to $\mathrm{n}=$ 2. These successive transitions of the mode can be due to the radial extension of the frequency accumulation point at the stronger helical core $\left(\delta_{H}=0.7\right)$, as they are not present for the lower helical excursion of $\left(\delta_{H}=0.37\right)$ as shown in figure 14 . 


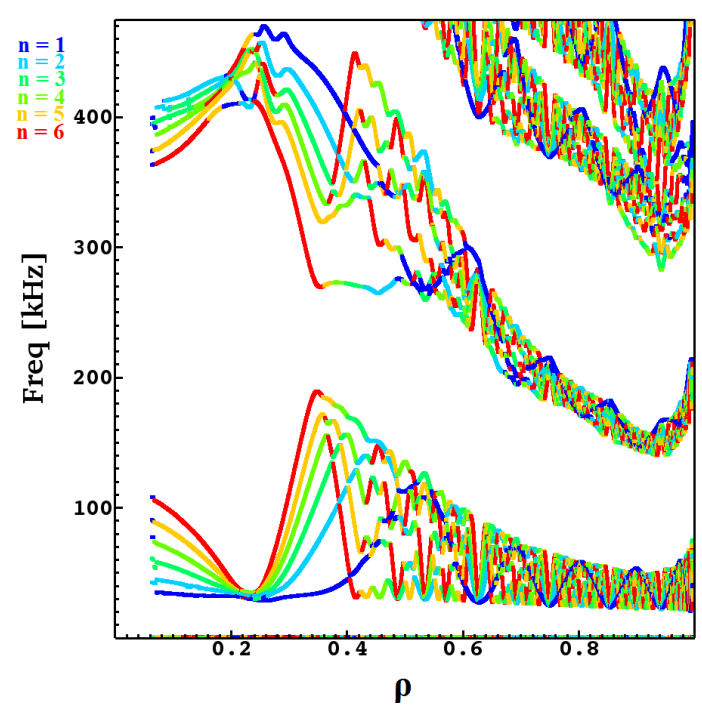

Figure 15: Alfvén continuum structures with $3 \mathrm{D}$ helical core equilibrium having $\delta_{H}=0.7$ in discharge \#20488, where pressure is scaled to 1.75 times

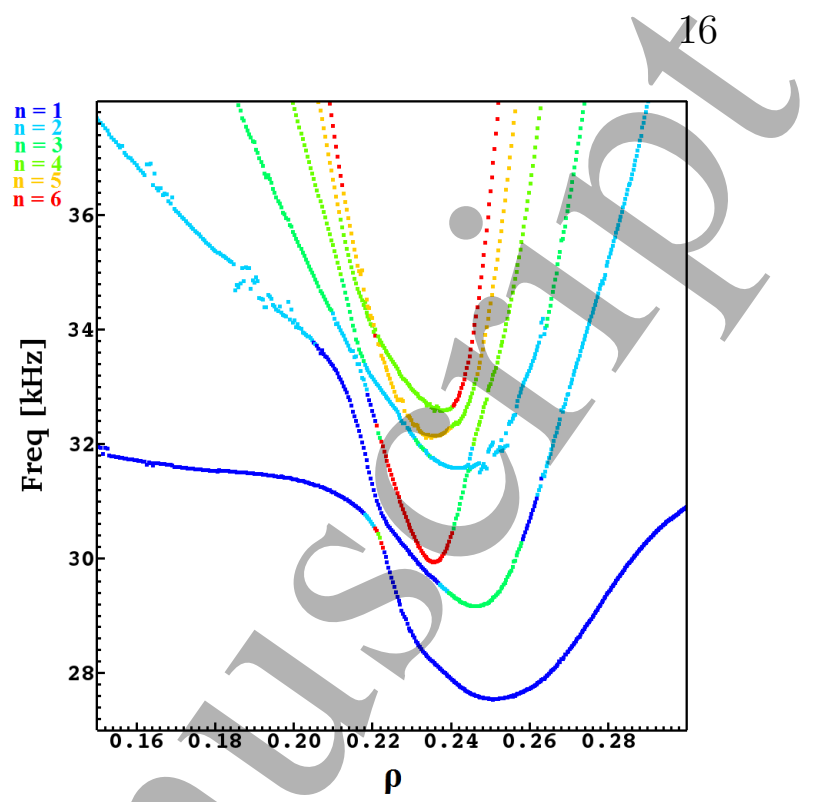

Figure 16: Zoom of Alfvén continua in Figure 15. Different colors represent the corresponding toroidal modes number (n)

The dependence of frequency/splitting around $\rho \approx 0.23$ on the helical core size is shown in Figure 17. It shows that splitting in frequency $(\Delta f)$ increases with the helical core size. It is very low, around $0.5 \mathrm{kHz}$ for small $\delta_{H}=0.22$ values and reaches $5 \mathrm{kHz}$ for the maximum helical core size of $\delta_{H}=0.7$.

Around the frequency splitting accumulation point, the radial variation of the Alfvén continuum extrema remains unchanged at the lower values of the helical core size $\delta_{H}$ as shown in Figure 18. However a small increase of $\rho=0.025$ has been noticed, which suggests that frequency splitting appears with a very small radial excursion of continua. For the maximum achieved helical core excursion $\left(\delta_{H}=0.7\right)$, a small difference of $\rho=0.016$ between the continua minimum and maximum frequencies shows that the frequency splitting is accompanied with a small movement of the accumulation point in radius.

Alfvén continuum calculations for the different levels of the helical core equilibria have been performed. The dependence of the Alfvén frequency gaps on $\delta_{H}$, formed by the extrema of $n=6$ continua with helical equilibria, around the plasma axis and at two further out radial locations is shown in Figure 19. Alfvén gaps at these three $\rho$ values show a decreasing tendency for further increases in helical core strength beyond $\delta_{H}=0.37$. Around the plasma axis $(\rho \approx 0)$, the continuum gaps are narrower than the gaps around $\rho \approx 0.23$ and wider than the gaps $\rho \approx 0.35$. Alfvén gaps for $\rho \approx 0$ at $\delta_{H}=0$ and $\delta_{H}=0.37$ are comparable having around $280 \mathrm{kHz}$ frequency and for the maximum helical excursion $\delta_{H}=0.7$, the gap is $255 \mathrm{kHz}$.

At the frequency splitting radial location $\rho \approx 0.23$, the Alfvén gap is larger for the axisymmetric case $\left(\delta_{H}=0\right)$ having a frequency of $420 \mathrm{kHz}$ as compared with the 
Helical Core paper

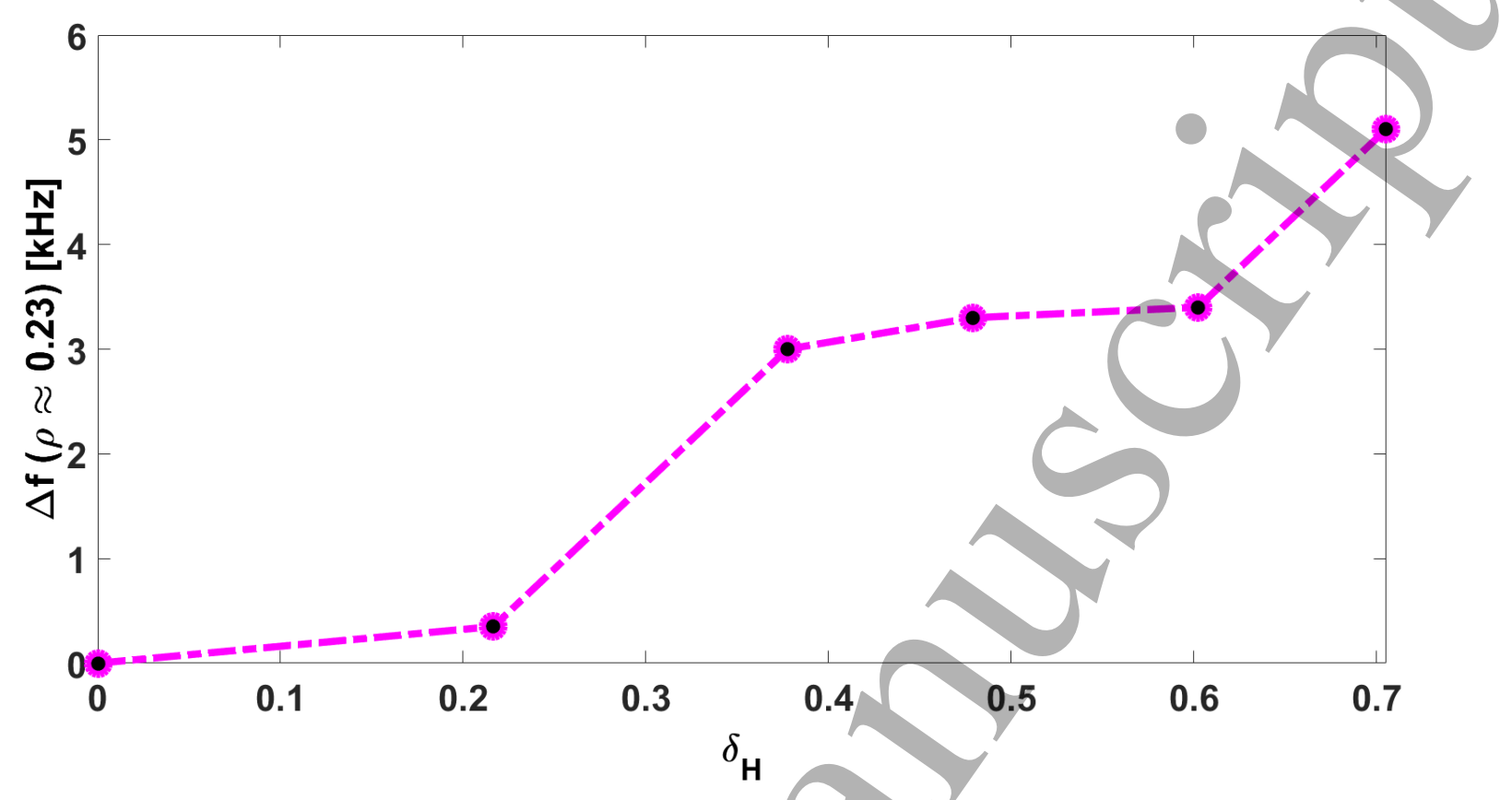

Figure 17: Alfvén continuum frequency splitting with helical core size around $\rho \approx 0.23$ when toroidal mode numbers $n=1-6$ have been taken into account in the calculations. If a larger number of n's had been included in the calculations, the $\Delta f$ could have been different.

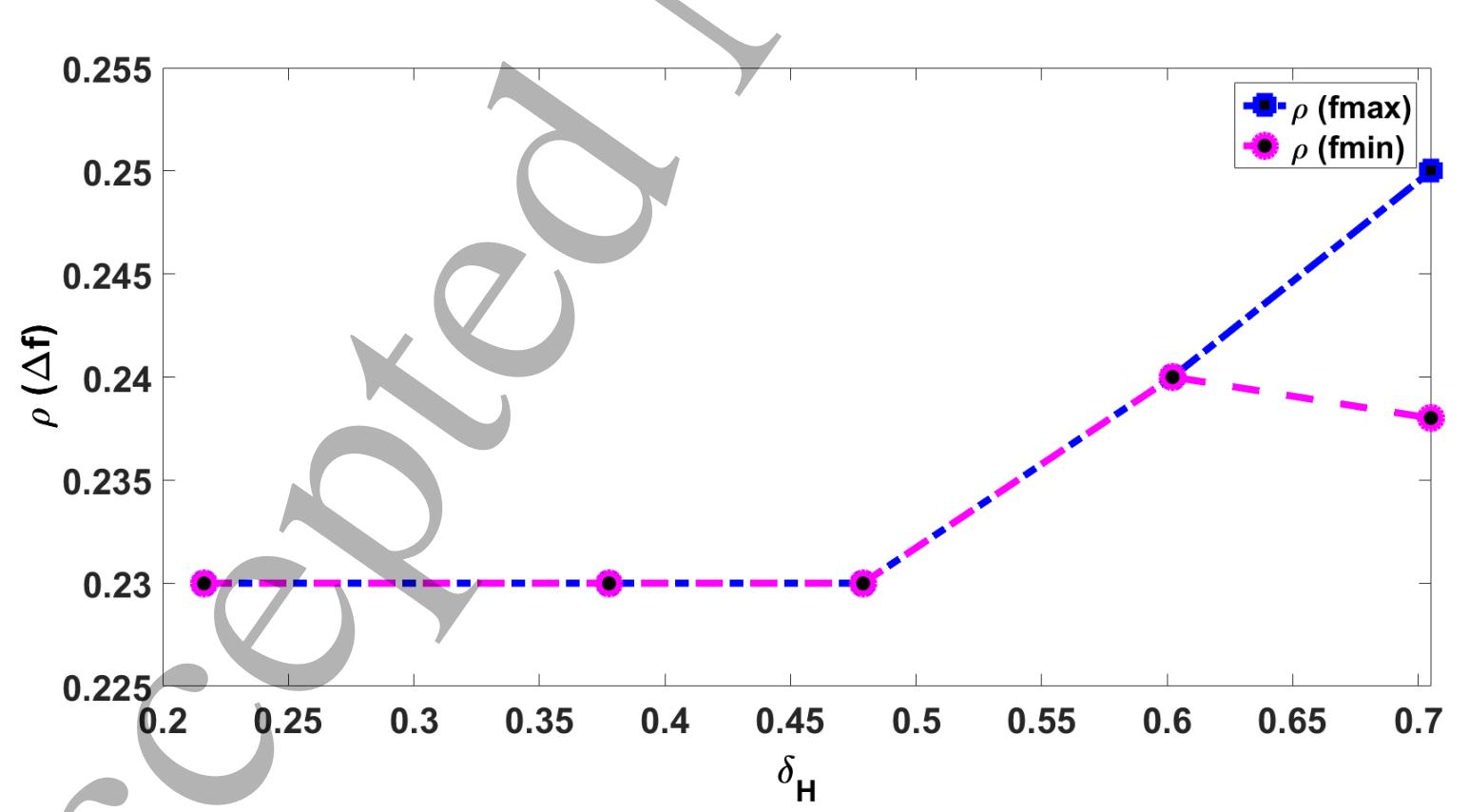

Figure 18: Radial variation of the frequency splitting accumulation point with helical core excursion

increasing values of $\delta_{H}$. The Alfvén gap around $\rho \approx 0.23$ decreases with an increase in $\delta_{H}$ $=0.37$ to 0.7 and reaches to $365 \mathrm{kHz}$ for largest helical core size. However for $\delta_{H}=0.22$ 


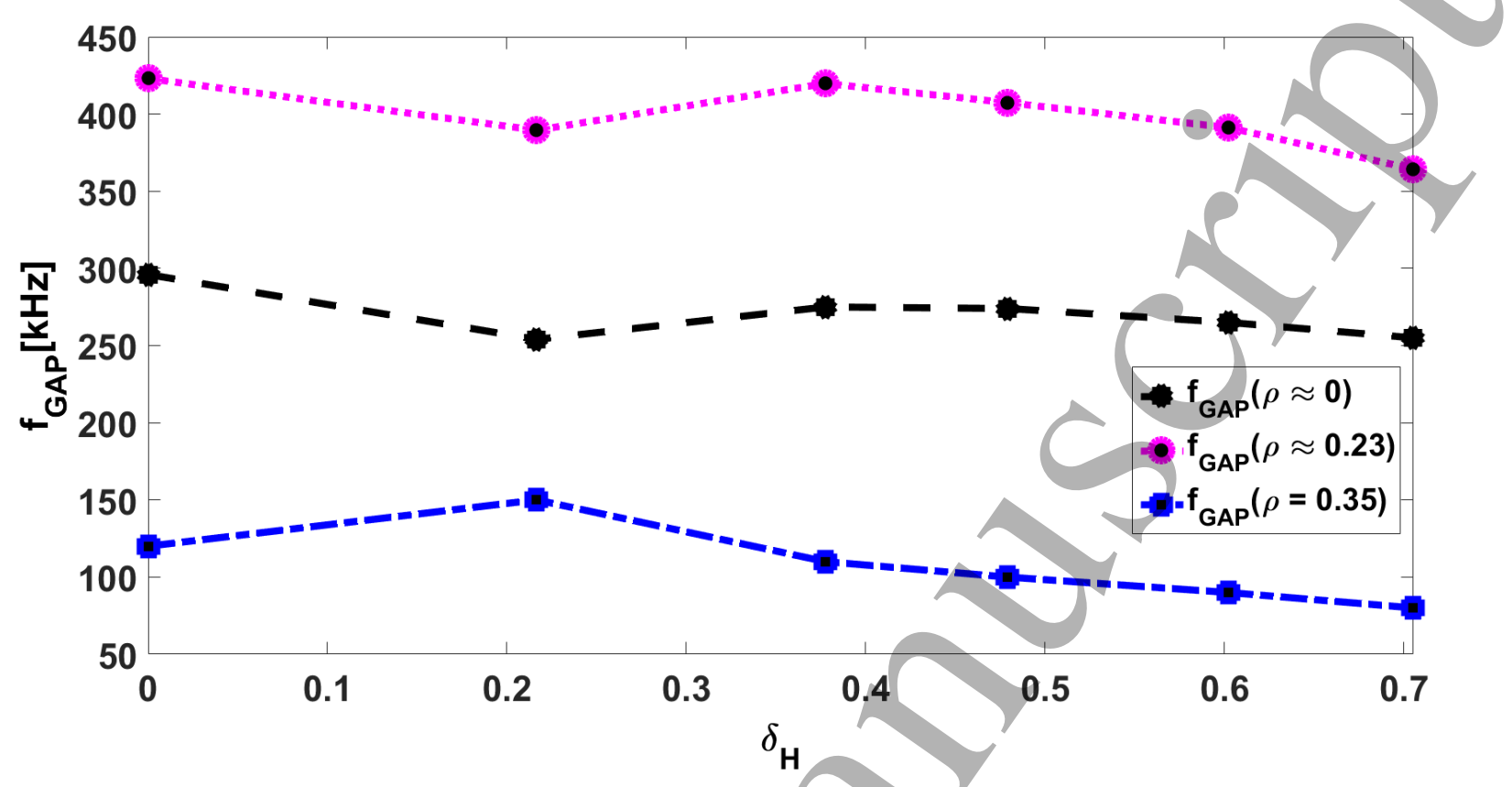

Figure 19: Alfvén frequency gap variation with the helical core size at three different radial locations

the gap is relatively smaller. It is also noticeable that at $\rho \approx 0.23$, the continuum gaps at $\delta_{H}=0$ and $\delta_{H}=0.37$ are comparable. The decrease in the Alfvén frequency gap has been encountered for higher values of $\rho$, at the radial location $\rho \approx 0.35$ the frequency gap decreases around in the range of $126 \mathrm{kHz}$ for the axisymmetric case. Contrary to the gap around $\rho \approx 0.23$, the Alfvén gap around $\rho \approx 0.35$ follows a decreasing trend from $\delta_{H}=0.22$ to 0.7 and reaches to $80 \mathrm{kHz}$ for the maximum helical core size $\delta_{H}=0.7$. In this case it is also noticeable, the Alfvén gaps at $\delta_{H}=0$ and $\delta_{H}=0.37$ are not alike as they were in the case of $\rho \approx 0.23$. The lower maxima of continuum structures for the formation of these gaps remained constant around $190 \mathrm{kHz}$ except for $\delta_{H}=0.22$ at which it decreased to $160 \mathrm{kHz}$. The gaps calculated at $\delta_{H}=0.22$ correspond to the twice the experimental plasma pressure. The increased plasma pressured decreases helical core excursion and hence the Alfvén gaps at all three radial locations. These results show that the Alfvén frequency gap is strongly influenced by the helical core size and also varies along the minor radius. Close to the plasma axis $\rho \approx 0$, the gap is smaller than at $\rho \approx 0.23$ and as $\rho$ increases from $\rho \approx 0.23$ to 0.4 the Alfvén frequency gap also decreases as shown in Figures 11, 13 and 15.

In these results, three major gaps have been found as characterized by their frequencies. In low frequency region the gap is identified with the BAE gap which is generated by the plasma compressibility and geodesic curvature. In between the two identical toroidal mode numbers, a wider gap has been found due to the adjacent poloidal mode couplings, therefore it is called TAE gap. At relative farther radial location and higher frequency a gap also forms, which is identified as the EAE gap. 


\section{Helical Core paper}

These results show that the TAE gap is the largest gap and has its maximum value around the frequency accumulations point $\rho \approx 0.23$ and it decreases at the outer radii. Thus it is expected that not only the BAE physics is modified but also the TAE (odd and even) and EAE mode frequencies which will be investigated elsewhere.

In comparison with $2 \mathrm{D}$ results given in Figures 11 and 12, a distortion in the continuum structures $\delta f_{A C}$ around $\rho \approx 0.35$ has been noticed (Figures 13 and 15), which appears only when the helical core $3 \mathrm{D}$ equilibrium is employed. We have inferred $\delta f_{A C}$ as conversion of the mode from one toroidal mode number to another; this generates localised gaps between the different continuum spectra along the radius starting from $\rho \approx 0.35$. Distortion in the Alfvén continuum provides an information about the formation of the localised gaps between different continua and the mode conversion which are absent in the axisymmetric calculations.

The maximum radial extent to which the effect of $\delta f_{A C}$ is modelled with the helical excursion $\delta_{H}$ is shown in figure 20. This shows, for smaller helical core $\left(\delta_{H}=0.22\right)$, the distortion in continua ranges up to $\rho \approx 0.42$ staring from $\rho \approx 0.35$ and it reaches up to $\rho \approx 0.56$ (figure 15 ) for the maximum achieved helical core size. It generates localised frequency gaps in radius between the different branches of continua. These are Helical Alfvén Eigenmode (HAE) gaps since they emerge due to the coupling of continuum branches from different n's. Up to $\delta_{H}=0.7$, the radial extension around $0.35 \leq \rho \leq 0.56$ of the HAE gaps between different continua suggests that they are radially extended modes. At $\delta_{H}=0.7$, radially localized closed HAE gaps also appear around $\rho \approx 0.56$.

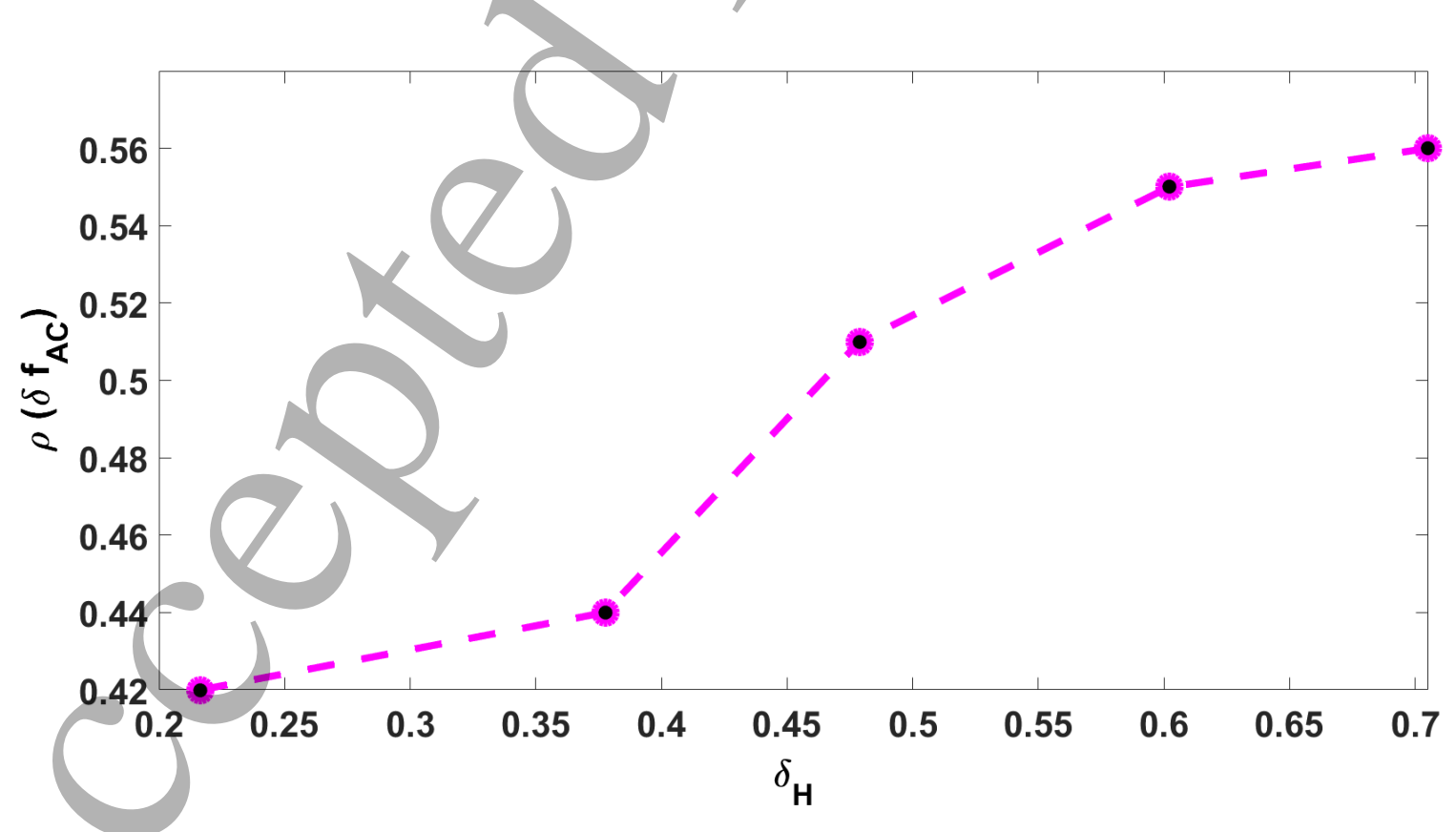

Figure 20: Variation in the radial extent of the Alfvén continuum distortion with helical core size

As per HAE definition [37], $n$ couples to $n \pm \delta_{n} N_{f p}$ and $m$ with $m \pm \delta_{m}$, where $n$ and 


\section{Helical Core paper}

$m$ are toroidal and poloidal mode numbers, $N_{f p}$ is field period and $\delta_{n}, \delta_{m}$ are integer mode displacements, therefore the coupling between successive n's is possible; this leads to open gaps between different $n$ continua. In our calculations, for a significant helical excursion of $\delta_{H}=0.7$, initially an $n=6$ mode (in red) converts to $n=5$ (yellow) and then $n=4$ (green) and generates a radially extended gap with the internal mode $n=5$ frequency (yellow) which then converts to $n=6$ (red), following to $n=4$ (green) and back to $n=5$ as function of radius. These gaps are limited in radial extent associated with the radial extension of $q=1$ rational surfaces as shown in figure 3.

Effects of the 3D density inhomogeneities on the Alfvén spectrum for tokamak plasmas [43] lead to the coupling of different $n$ Alfvén continuum is possible. Specifically, when the toroidal inhomogeneity is prominent these couplings produce new gaps where the continua of the two toroidal harmonics were degenerate in the homogeneous case. Similarly, for in helical equilibria the continuum spectrum is distorted, with the generation of additional gaps caused by the coupling of successive $n$ in radius.

This splitting of Alfvén continua with helical core size is helpful to explain the experimental observations: first one has to note that lower mode numbers have lower accumulation points for the acoustic-Alfvén modes (see e.g. fig 13 of [17]). However, the BAE modes observed in the experiment show the inversion of the mode numbers from the kinetic simulations [16]. Towards the end of the sawtooth cycle the splitting of the modes remains in the model whereas it disappears experimentally (see $t=1.98$ of fig 7 in reference [17]). Before the crash, the core pressure is at its maximum, and despite some sawtooth precursors it remains large in the last $10 \mathrm{~ms}$ before the crash. The modification of the BAE accumulation points due to the helical core suggest that the splitting should decrease since it cancels the 'inverse diamagnetic' splitting discussed in reference [17]. In addition, also a small radial shift of the BAE accumulation point due the helical core is observed (see fig. 16 [17]), changing the kinetic BAE accumulation point because of radial profile (mainly $T_{i}$ and $T_{e}$ ) effects. Thus, the combination of helical core $(\sim 3-5 \mathrm{kHz}$ shift $)$ and diamagnetic downshift may go hand in hand to explain the experimental observations. Very demanding global kinetic 3D calculations would be needed to investigate this question more quantitatively.

\section{Summary and conclusions}

Investigations for the Alfvén continuum spectra of helically distorted MHD equilibrium have been carried out for improved understanding of observed low-frequency Alfvénic modes in tokamak plasmas. First, we have carried out the bifurcated MHD equilibrium investigations for the tokamak plasmas using a 3D numerical tool (VMEC). Identical sets of parameters have been employed in the reconstructions of axisymmetric (2D) and helical core (3D) equilibria, with the only difference being a small seed perturbation in the magnetic axis. The formation of the helical core has been associated with a saturated internal-kink $\mathrm{m}=1, \mathrm{n}=1$ mode [14] which determines its excursion level. In previous studies [17], a kinetic model has been applied to the experimental findings. We find that 


\section{Helical Core paper}

near rational surfaces having $q=1$, the Alfvén continuum splits into multiple frequencies and the continuum of $n$ couples with that of $n-1$. However, despite the promising results, the kinetic model [17] alone could not well describe the remaining differences of the 2 to $5 \mathrm{kHz}$ in the frequency splitting. Results for the Alfvén continuum calculations obtained with the extended STELLGAP solver including the 3D effects of helical core equilibria, explain these few $\mathrm{kHz}$ differences in the frequency splitting, and confirm the frequency splitting phenomenon around $q=1$ rational surfaces. The continua obtained with gaps opened by the plasma compressibility and geodesic curvature are in the range of frequencies of Alfvénic (BAE) and mixed acoustic-Alfvénic (BAAE) modes, which support the experimental findings of low-frequency modes.

The sensitivity studies with pressure variation show that the helical excursion of the equilibrium increases with increasing plasma pressure and eventually it drops at extreme pressures due to the fixed boundary equilibrium calculations. Fixed boundary reconstruction has been considered sufficient in these calculations because of the desired plasma pressure scaling. The continuum results show that the frequency splitting around the accumulation point also increases with increases in the helical excursion. The accumulation point does not vary in radius with the plasma pressure. The distortion in the continua along the radial extent starting from $\rho \approx 0.35$ has been found, which is also an effect from the helical core equilibria.

Finally, it is concluded that these new results are comparable with the experimental findings discussed in [17], with the confirmation of frequency splitting in Alfvén continuum via coupling of adjacent $n$ continua around an accumulation point. Additionally, the issue of few $\mathrm{kHz}$ differences in the frequency splitting is addressed and explained by including 3D effects. Furthermore, the formation of additional lowfrequency gaps due to the coupling of acoustic-Alfvén waves are also addressed in our calculations.

\section{Acknowledgements}

This work has been carried out within the framework of the EUROfusion Consortium and has received funding from the Euratom research and training programme 2014-2018 and 2019-2020 under grant agreement No 633053. The views and opinions expressed herein do not necessarily reflect those of the European Commission. Allah Rakha is grateful for an AGAUR FI predoctoral grant to support his PhD studies and a Severo Ochoa (SO) mobility grant to support his research stay at Max Planck Institute for Plasma Physies (IPP) Garching, Germany. We would also like to thank Dr.Erika Strumberger for her helpful advice concerning the use of VMEC code for the helical core equilibrium reconstruction.

\section{References}

[1] Chen L. and Zonca F. 2007 Nucl. Fusion 47 S727 


\section{Helical Core paper}

[2] Chen L. 1994 Phys. Plasmas 11519

[3] Cheng C. Z., Chen L. and Chance M.S. 1985 Ann. Phys. 16121

[4] Hasegawa A. and Chen L. 1974 Phys. Rev. Lett. 32454

[5] Chen L. and Hasegawa A. 1974 Phys. Fluids 171399

[6] Biancalani A. et al 2010 Phys. Plasmas 17122106

[7] Cooper W. A. et al 2011 Nucl. Fusion 51072002

[8] Lorenzini R. et al 2009 Nature Physics 5570

[9] Cooper W. A. et al 2013 Nucl. Fusion 53073021

[10] Joffrin E. 2005 Nucl. Fusion 45626

[11] Bonofiglo P. J. et al 2019 Phys. Plasmas 26022502

[12] Chapman I. T. et al 2010 Nucl. Fusion 50045007

[13] Pfefferlé D. et al 2014 Nucl. Fusion 54064020

[14] Lazarus E. A. et al 2006 Plasma Phys. Control. Fusion 48 L65

[15] Camenen Y. et al 2007 Nucl. Fusion 47586

[16] Lauber Ph. et al 2009 Plasma Phys. Control. Fusion 51124009

[17] Curran D. et al 2012 Plasma Phys. Control. Fusion 54055001

[18] Zonca F., Chen L. and Santoro R.A. 1996 Plasma Phys. Control. Fusion 382011

[19] Lauber Ph. 2013 Physics Reports 53333

[20] Lauber Ph. et al 2007 J. Comput. Phys. 226447

[21] Hirshman S. P. and Whitson J. C. 1983 Phys. Fluids 263553

[22] Deng C. B. et al 2009 Phys. Rev. Lett. 103025003

[23] Spong D. A. et al 2010 Contrib. Plasma Phys. 50708

[24] Rakha A.et al 2019 Nucl. Fusion 59056002

[25] Spong D. A. et al 2017 Nucl. Fusion 57086018

[26] Cook C. R. et al 2016 Plasma Phys. Control. Fusion 58054004

[27] Spong D. A. 2013 Nucl. Fusion 53053008

[28] Fesenyuk O.P., Kolesnichenko Ya.I., Wobig H. and Yakovenko Yu.V. International Workshop "Innovative Concepts and Theory of Stellarators", Kyiv, Ukraine, 28-31 May 2001, Kyiv, ISSN 1606-6723, p. 105 (2001)

[29] Könies A. 2007 A code for the calculation of kinetic Alfvén waves in three-dimensional Geometry 10th IAEA TM on Energetic Particles in Magnetic Confinement Systems

[30] Könies A. and Eremin D.2010 Phys. Plasmas 17012107

[31] Lauber Ph. et al 2012 Nucl. Fusion 52094007

[32] Kramer G.J. et al 2016 Plasma Phys. Control. Fusion 58085003

[33] Cooper W. A. et al 2010 Phys. Rev. Lett. 105035003

[34] Cooper W. A. et al 2011 Plasma Phys. Control. Fusion 53074008

[35] Strumberger E., Günter S. and Tichmann C. 2014 Nucl. Fusion 54064019

[36] Cooper W. A. et al 2011 Plasma Phys. Control. Fusion 53024002

[37] Spong D. A., Sanchez R. and Weller A. 2003 Phys. Plasmas 103217

[38] Chu M. et al 1992 Phys. Fluids B 43713

[39] Fesenyuk O. P., Kolesnichenko Ya. I., Wobig H. and Yakovenko Yu. V. 2002 Phys. Plasmas 91589

[40] Cheng C. Z. and Chance M. S. 1986 Phys. Fluids B 29, 3695

[41] Wingen A. et al 2018 Nucl. Fusion 58036004

[42] Kobuchi T. et al 2006 Plasma Phys. Control. Fusion 48789

[43] Oliver H.J.C. et al 2018 Modification of the Alfvén spectrum by 3D density inhomogeneities 45th EPS Conference on Plasma Physics, Prague, Czech Republic 2018.

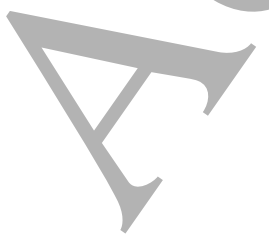

